\title{
Theory of a Slotted-Sphere Antenna Immersed in a Compressible Plasma. ${ }^{1} \quad$ Part II
}

\author{
James R. Wait \\ Contribution From the Central Radio Propagation Laboratory, National Bureau of Standards, \\ Boulder, Colo.
}

(Received May 20, 1964)

\begin{abstract}
Radiation from a source immersed in a compressible and lossy electron plasma is considered. The model is a dielectric-coated conducting sphere which is excited uniformly by an annular slot. As in part I, it is assumed that the fields in the isotropic plasma are governed by Maxwell's equations in combination with (single fluid) continuum theory of fluid dynamies. Formulas are developed which should be suitable for computing the admittance of the annular slot.
\end{abstract}

\section{Introduction}

In a paper of the same title (but denoted part I), the theory for a slotted-sphere antenna in a lossless and compressible plasma was considered. In this paper (denoted part II), the surrounding plasma medium is allowed to have losses in the form of collisions between electrons and the heavy particles. However, the (electron) acoustic waves are again assumed to propagate with a velocity $u$ without attenuation. Another modification of the earlier analysis is to allow for the existence of a dielectric coating surrounding the slotted sphere. As an idealization of what may be expected in nature, the sheath is regarded as a lossless dielectric coating which is perfectly rigid for the (electron) acoustic waves. While such a model is highly idealized, it does permit an analysis to be carried out in a relatively tractable manner. Furthermore, by preventing the lossy material (i.e., the plasma) from being in direct contact with the sphere, a meaningful power calculation may be carried out. Also, the presence of the coating allows one to say something about how the susceptance of the slotted sphere is modified by the presence of the plasma.

\section{Formulation}

As in part I, the isotropic plasma is regarded as a one-component electron fluid. The average number density of the electrons is denoted $n_{0}$, the pressure deviation from the mean is $p$, the electric field is $\vec{E}$, and the magnetic field is $\vec{H}$. The collisions between the electrons and the heavy particles are assumed to be accounted for by an energy-independent collision frequency $\nu$.

The linearized equation of motion is now given by

$$
m n_{0}\left(\nu+\frac{\partial \vec{v}}{\partial t}\right)=n_{0} e \vec{E}-\nabla p
$$

\footnotetext{
1 The work was supported by the Air Force Cambridge Research Laboratories, Bedford, Mass., PRO-62-201.
} 


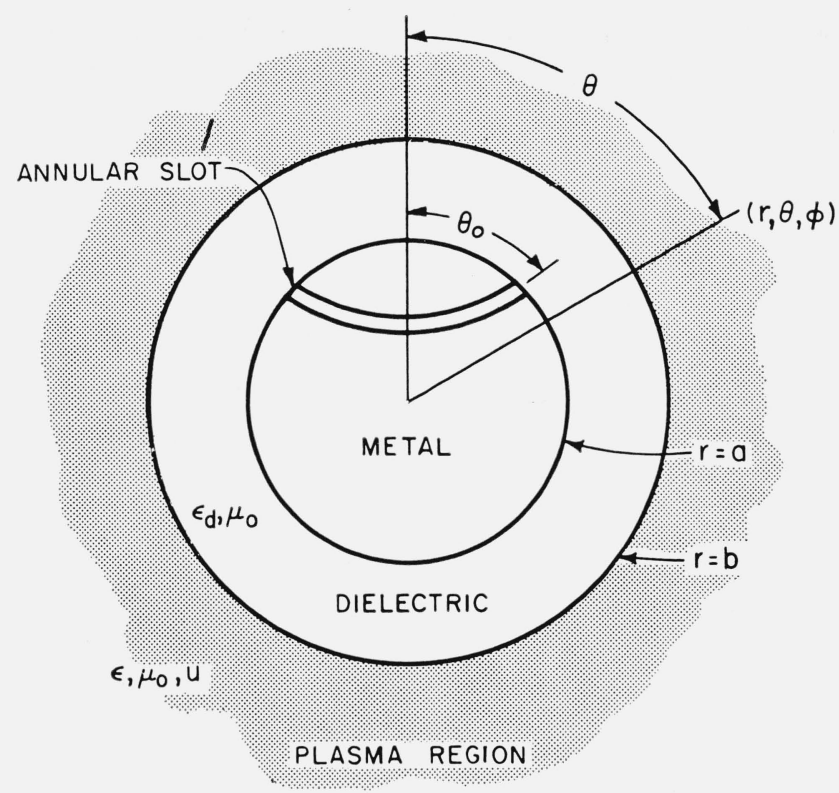

FIGURE 1. Slotted-sphere antenna, with a dielectric sheath, immersed in a compressible plasma.

where $e$ and $m$ are the charge and mass of the electrons. The usual linearized equation of continuity, combined with the equation of state, is

$$
u^{2} m n_{0} \nabla \cdot \vec{v}=-\frac{\partial p}{\partial t}
$$

where, as mentioned, $u$ is the velocity of sound in the electron gas.

The geometrical configuration is shown in figure 1 with respect to a spherical coordinate system $(r, \theta, z)$. The slotted sphere is bounded by the surface $r=a$ and it is perfectly conducting except for a narrow annular slot at $\theta=\theta_{0}$. The sphere is encased by a rigid dielectric covering whose outer surface is at $r=b$. The dielectric constant of this sheath is $\epsilon_{d}$ and the permeability is $\mu_{0}$, which is taken to be the same as free space.

The homogeneous compressible plasma region is taken to occupy the space exterior to the surface $r=b$. The equations in motion in spherical coordinates are now written

$$
(\nu+i \omega) m n_{0} v_{r}=n_{0} e E_{r}-\frac{\partial p}{\partial r},
$$

and

$$
(\nu+i \omega) m n_{0} v_{\theta}=n_{0} e E_{\theta}-\frac{1}{r} \frac{\partial p}{\partial \theta}
$$

where, because of symmetry, $v_{\phi}, E_{\phi}$, and $\partial p / \partial \phi$ are all zero. The corresponding form of the continuity equation is given by

$$
\frac{u^{2} m n_{0}}{r^{2} \sin \theta}\left[\sin \theta \frac{\partial}{\partial r}\left(r^{2} v_{r}\right)+r \frac{\partial}{\partial \theta}\left(v_{\theta} \sin \theta\right)\right]=-i \omega p
$$


On combining (3) and (4) with Maxwell's equations, one is led readily to the set

$$
\begin{gathered}
E_{\theta}=-\frac{1}{i \epsilon \omega} \frac{1}{r} \frac{\partial}{\partial r}\left(r H_{\phi}\right)+\frac{e}{(\nu+i \omega) m i \epsilon \omega r} \frac{\partial p}{\partial \theta}, \\
E_{r}=\frac{1}{i \epsilon \omega} \frac{1}{r \sin \theta} \frac{\partial}{\partial \theta}\left(\sin \theta H_{\phi}\right)+\frac{e}{(\nu+i \omega) m i \epsilon \omega} \frac{\partial p}{\partial r}, \\
v_{\theta}=\frac{-e}{(i \epsilon \omega)(\nu+i \omega) m} \frac{1}{r} \frac{\partial}{\partial r}\left(r H_{\phi}\right)-\frac{\epsilon_{0}}{(\nu+i \omega) n_{0} \epsilon m r} \frac{\partial p}{\partial \theta}, \\
v_{r}=\frac{e}{(i \epsilon \omega)(\nu+i \omega) m} \frac{1}{r \sin \theta} \frac{\partial}{\partial \theta}\left(\sin \theta H_{\phi}\right)-\frac{\epsilon_{0}}{(\nu+i \omega) n_{0} \epsilon m} \frac{\partial p}{\partial r},
\end{gathered}
$$

where

$$
\frac{\epsilon}{\epsilon_{0}}=1+\frac{\omega_{0}^{2}}{i \omega(\nu+i \omega)} \text { with } \omega_{0}^{2}=\frac{n_{0} e^{2}}{m \epsilon_{0}} .
$$

In the above, $\epsilon$ is the (complex) dielectric constant of the plasma, $\epsilon_{0}$ is the dielectric constant of free space, and $\omega_{0}$ is the (angular) electron plasma frequency.

Following the procedures in the earlier papers [Wait, 1964a and 1964b], it is not difficult to show that within the compressible plasma medium

and

$$
r \frac{\partial^{2}}{\partial r^{2}}\left(r H_{\phi}\right)+\frac{\partial}{\partial \theta}\left[\frac{1}{\sin \theta} \frac{\partial}{\partial \theta}\left(\sin \theta H_{\phi}\right)\right]-\gamma_{e}^{2} r^{2} H_{\phi}=0
$$

$$
\frac{\partial}{\partial r}\left(r^{2} \frac{\partial p}{\partial r}\right)+\frac{1}{\sin \theta} \frac{\partial}{\partial \theta}\left(\sin \theta \frac{\partial p}{\partial \theta}\right)-\gamma_{p}^{2} r^{2} p=0
$$

where

$$
\gamma_{e}=i\left(\epsilon \mu_{0}\right)^{\frac{1}{2}} \omega=\frac{\omega}{c}\left(\frac{\epsilon}{\epsilon_{0}}\right)^{\frac{1}{2}}
$$

and

$$
\gamma_{p}=i \frac{\omega}{u}\left(1+\frac{\nu}{i \omega}\right)^{\frac{1}{2}}\left(\frac{\epsilon}{\epsilon_{0}}\right)^{\frac{1}{2}}=\frac{i}{u}\left[\left(\omega^{2}-\omega_{0}^{2}\right)-i \omega \nu\right]^{\frac{1}{2}}
$$

The radicals in the above expressions are chosen so that the real parts of $\gamma_{e}$ and $\gamma_{p}$ are positive.

Appropriate solutions of (10) and (11) are

$$
H_{\phi}=\sum_{n=1}^{\infty} c_{n} \frac{\hat{K}_{n}\left(\gamma_{e} r\right)}{\gamma_{e} r} P_{n}^{\prime}(\cos \theta),
$$

and

$$
p=\sum_{n=1}^{\infty} a_{n} \frac{\hat{K_{n}}\left(\gamma_{p} r\right)}{\gamma_{p} r} P_{n}(\cos \theta),
$$

where $a_{n}$ and $c_{n}$ are coefficients, $\hat{K}_{n}$ is a modified spherical Bessel function defined before [Wait, $1964 \mathrm{a}$ and $1964 \mathrm{~b}]$, and $P_{n}$ is the Legendre polynomial, while $P_{n}^{\prime}=\partial P_{n} / \partial \theta$. For the boundary condition $v_{r}=0$ at $r=b$, it also follows from (9) that

$$
\frac{a_{n}}{c_{n}}=-\frac{r_{0}(n+1) n_{0} e}{\gamma_{p} \epsilon_{0} b i \omega} \frac{\left[\hat{K}_{n}\left(\gamma_{e} b\right)\right] /\left(\gamma_{e} b\right)}{\left[\frac{\hat{K}_{n}^{\prime}\left(\gamma_{p} b\right)}{\gamma_{p} b}-\frac{\hat{K}_{n}\left(\gamma_{p} b\right)}{\left(\gamma_{p} b\right)^{2}}\right]} \cdot
$$


In order to solve the complete boundary value problem, it is desirable to use the expression for the impedance for mode $n$ at the boundary $r=b$. This is defined by writing the tangential fields in the form

$$
H_{\phi}(b, \theta)=\sum_{n=1}^{\infty} c_{n} \frac{\hat{K}_{n}\left(\gamma_{e} b\right)}{\left(\gamma_{e} b\right)} P_{n}^{\prime}(\cos \theta)
$$

and

$$
E_{\theta}(b, \theta)=\sum_{n=1}^{\infty} c_{n} Z_{n} \frac{\hat{K}_{n}\left(\gamma_{e} b\right)}{\gamma_{e} b} P_{n}^{\prime}(\cos \theta) .
$$

An explicit expression for $Z_{n}$ may be found from (6), along with (14), (15), and (16). Thus,

where

$$
Z_{n}=Z_{0 n}\left(1-\delta_{n}\right)
$$

and

$$
\delta_{n}=i n(n+1) \frac{\omega_{0}^{2}}{\omega(\nu+i \omega)\left(\gamma_{e} b\right)\left(\gamma_{p} b\right)} \frac{\hat{K}_{n}\left(\gamma_{e} b\right)}{\hat{K}_{n}^{\prime}\left(\gamma_{e} b\right)} \frac{\hat{K}_{n}\left(\gamma_{p} b\right)}{\left[\hat{K}_{n}^{\prime}\left(\gamma_{p} b\right)-\frac{\hat{K}_{n}\left(\gamma_{p} b\right)}{\left(\gamma_{p} b\right)}\right]}
$$

$$
Z_{0 n}=-\eta_{e} \frac{\hat{K}_{n}^{\prime}\left(\gamma_{e} b\right)}{\hat{K}_{n}\left(\gamma_{e} b\right)} \text { with } \eta_{e}=\gamma_{e} /(i \epsilon \omega)=\left(\mu_{0} / \epsilon\right)^{1 / 2}
$$

\section{Dielectric Region}

In the region $a<r<b$, the acoustic-type wave does not propagate. A suitable representation for the tangential fields is readily found. Thus,

and

$$
H_{\phi}=\sum_{n=1}^{\infty}\left[A_{n} \frac{\hat{K}_{n}(\gamma r)}{\gamma r}+B_{n} \frac{\hat{I}_{n}(\gamma r)}{\gamma r}\right] P_{n}^{\prime}(\cos \theta),
$$

$$
E_{\theta}=-\eta \sum_{n=1}^{\infty}\left[A_{n} \frac{\hat{K}_{n}^{\prime}(\gamma r)}{\gamma r}+B_{n} \frac{\hat{I}_{n}^{\prime}(\gamma r)}{\gamma r}\right] P_{n}^{\prime}(\cos \theta),
$$

where $\gamma=i\left(\epsilon_{d} \mu_{0}\right)^{1 / 2} \omega$ and $\eta=\left(\epsilon_{d} / \mu_{0}\right)^{1 / 2}$ and where $A_{n}$ and $B_{n}$ are coefficients yet to be determined. The functions $\hat{K}_{n}(z)$ and $\hat{I}_{n}(z)$ are an independent solution of the differential equation

$$
\frac{d^{2} w}{d z^{2}}=\left[1+\frac{n(n+1)}{z^{2}}\right] w
$$

and they are defined in terms of cylindrical-type Bessel functions by the relations

and

$$
\hat{K}_{n}(z)=(2 z / \pi)^{\frac{1}{2}} K_{n+1 / 2}(z),
$$

$$
\hat{I}_{n}(z)=(\pi z / 2)^{\frac{1}{2}} I_{n+1 / 2}(z)
$$

The conditions imposed on (22) and (23) are that they should be identical to (17) and (18) at $r=b$. Also, at $r=a$, the tangential electric field is to be specified. Thus, it is convenient to write

$$
E_{\theta}(a, \theta)=-\frac{\eta}{\gamma a} \sum_{n=1}^{\infty} e_{n} P_{n}^{\prime}(\cos \theta)
$$


where, as a result of orthogonality,

$$
e_{n}=-\left(\frac{\gamma a}{\eta}\right) \frac{(2 n+1)}{2 n(n+1)} \int_{0}^{\pi} E_{\theta}(a, \theta) P_{n}^{\prime}(\cos \theta)(\sin \theta) d \theta
$$

or

$$
e_{n}=-\left(\frac{\gamma}{\eta}\right) \frac{2 n+1}{2 n(n+1)} V_{0} P_{n}^{\prime}\left(\cos \theta_{0}\right) \sin \theta_{0}
$$

The coefficients may now be found from a straightforward algebraic process, to yield

$$
\begin{gathered}
c_{n}=\left(\gamma_{e} / \gamma\right) e_{n}\left[\hat{K}_{n}\left(\gamma_{e} b\right) \Lambda_{n}\right]^{-1}, \\
A_{n}=e_{n}\left[\hat{1_{n}^{\prime}}(\gamma b)+\left(Z_{n} / \eta\right) I_{n}(\gamma b)\right] / \Lambda_{n}, \\
B_{n}=-e_{n}\left[\hat{K}_{n}^{\prime}(\gamma b)+\left(Z_{n} / \eta\right) K_{n}(\gamma b)\right] / \Lambda_{n},
\end{gathered}
$$

where

$$
\Lambda_{n}=\left[\hat{l}_{n}^{\prime}(\gamma b) \hat{K}_{n}^{\prime}(\gamma a)-\hat{I}_{n}^{\prime}(\gamma a) \hat{K}_{n}^{\prime}(\gamma b)\right]+\left(Z_{n} / \eta\right)\left[\hat{l}_{n}(\gamma b) \hat{K}_{n}^{\prime}(\gamma a)-\hat{K}_{n}(\gamma b) \hat{l}_{n}^{\prime}(\gamma a)\right]
$$

As an immediate check on the results one may let $\operatorname{Re}(\gamma b) \rightarrow \infty$, whence

$$
A_{n} \rightarrow e_{n} \frac{1}{\hat{K}_{n}^{\prime}(\gamma a)} \text { and } B_{n} \rightarrow 0
$$

which corresponds to the situation where the slotted sphere is in an infinite homogeneous dielectric medium with electric constants $\epsilon_{d}$ and $\mu_{0}$. Another limiting case is to let $b \rightarrow a$, whence

$$
\Lambda_{n}=-Z_{n} / \eta \text { and } c_{n}=-\gamma_{e}(\eta / \gamma) e_{n} \hat{K}_{n}\left(\gamma_{e} a\right) / Z_{n}
$$

The limiting form of the magnetic field in the plasma is then given by

$$
H_{\phi}=-\frac{V_{0} \sin \theta_{0}}{2 \eta_{e} r} \sum_{n=1}^{\infty} \frac{(2 n+1)}{n(n+1)} \frac{\hat{K}_{n}\left(\gamma_{e} r\right)}{\hat{K}_{n}^{\prime}\left(\gamma_{e} a\right)} \frac{P_{n}^{\prime}\left(\cos \theta_{0}\right)}{1-\delta_{n}} P_{n}^{\prime}(\cos \theta),
$$

which is identical to (52) in the previous paper [Wait, 1964a] which is part I.

The tangential magnetic field at the interface between the dielectric and the plasma is conveniently written

$$
H_{\phi}(b, \theta)=-\frac{V_{0} \sin \theta_{0}}{2 \eta b} \sum_{n=1}^{\infty} \frac{(2 n+1)}{n(n+1)} \frac{1}{\Lambda_{n}} P_{n}^{\prime}\left(\cos \theta_{0}\right) P_{n}^{\prime}(\cos \theta),
$$

which has the required property that $\Lambda_{n} \rightarrow \hat{K}_{n}^{\prime}(\gamma a) / \hat{K}_{n}(\gamma b)$ when the plasma medium becomes a dielectric with electrical constants $\epsilon_{d}$ and $\mu_{0}$.

\section{Sheath Function $\Lambda_{n}$}

The influence of the dielectric sheath is determined mainly by the function $\Lambda_{n}$ for the modes of order $n$. Therefore, a discussion of this function is in order. The starting point is the development of the Bessel functions $\hat{I}_{n}(\gamma b)$ and $\hat{K}_{n}(\gamma b)$ as Taylor series about $\hat{I}_{n}(\gamma a)$ and $\hat{K}_{n}\left(\gamma_{a}\right)$, respectively. Denoting either of these functions by $w$, it follows that

$$
w(\gamma b)=w(\gamma a)+\Delta w^{\prime}(\gamma a)+\left(\Delta^{2} / 2\right) w^{\prime \prime}(\gamma a)+\left(\Delta^{3} / 6\right) w^{\prime \prime \prime}(\gamma a)+\left(\Delta^{4} / 24\right) w^{i v}(\gamma a)+\ldots,
$$


where $\Delta=\gamma(b-a)$. The higher derivatives may be reduced by noting that $w(z)$ satisfies the differential equation

$$
w^{\prime \prime}(z)=\left[1+\frac{n(n+1)}{z^{2}}\right] w(z)
$$

which enables one to write

$$
w^{\prime \prime \prime}(z)=\left[1+\frac{n(n+1)}{z^{2}}\right] w^{\prime}(z)-\frac{2 n(n+1)}{z^{3}} w(z),
$$

and

$$
w^{i v}(z)=\left\{\left[1+\frac{n(n+1)}{z^{2}}\right]^{2}-\frac{6 n(n+1)}{z^{4}}\right\} w(z)-\frac{4 n(n+1)}{z^{3}} w^{\prime}(z) .
$$

In general,

and

$$
w(\gamma b)=R_{n} w(\gamma a)+Q_{n} w^{\prime}(\gamma a),
$$

where

$$
w^{\prime}(\gamma b)=M_{n} w(\gamma a)+N_{n} w^{\prime}(\gamma a),
$$

$$
\begin{gathered}
R_{n}=1+\frac{\Delta^{2}}{2}\left[1+\frac{n(n+1)}{(\gamma a)^{2}}\right]-\frac{\Delta^{3}}{3}\left[\frac{n(n+1)}{(\gamma a)^{3}}\right]+\ldots, \\
Q_{n}=\Delta+\frac{\Delta^{3}}{6}\left[1+\frac{n(n+1)}{(\gamma a)^{3}}\right]-\frac{\Delta^{4}}{6}\left[\frac{n(n+1)}{(\gamma a)^{3}}\right]+\ldots \\
M_{n}=\Delta\left[1+\frac{n(n+1)}{(\gamma a)^{2}}\right]-\Delta^{2}\left[\frac{n(n+1)}{(\gamma a)^{3}}\right]+\frac{\Delta^{3}}{6}\left[\left(1+\frac{n(n+1)}{(\gamma a)^{2}}\right)^{2}-\frac{6 n(n+1)}{(\gamma a)^{4}}\right]+\ldots, \\
N_{n}=1+\frac{\Delta^{2}}{2}\left[1+\frac{n(n+1)}{(\gamma a)^{3}}\right]-\frac{\Delta^{3}}{3}\left[\frac{2 n(n+1)}{(\gamma a)^{3}}\right]+\ldots
\end{gathered}
$$

where

$$
\Delta=\gamma(b-a)=i\left(\epsilon_{d} \mu_{0}\right)^{1 / 2} \omega(b-a) .
$$

The expansions in ascending powers of $\Delta$ converge quite well for dielectric sheaths that are sufficiently thin (e.g., such that $|\Delta|<1$ ). As a result of the above basic definitions,

and

$$
\hat{I}_{n}(\gamma b) \hat{K}_{n}^{\prime}(\gamma a)-\hat{K}_{n}(\gamma b) \hat{I_{n}^{\prime}}(\gamma a)=-R_{n},
$$

$$
\hat{I}_{n}^{\prime}(\gamma b) \hat{K}_{n}^{\prime}(\gamma a)-\hat{K}_{n}^{\prime}(\gamma b) \hat{I}_{n}^{\prime}(\gamma a)=-M_{n}
$$

Thus,

$$
\Lambda_{n}=-\left[M_{n}+\frac{Z_{n}}{\eta} R_{n}\right]=-M_{n}+\frac{\eta_{e}}{\eta} \frac{\hat{K}_{n}^{\prime}\left(\gamma_{e} b\right)}{\hat{K}_{n}\left(\gamma_{e} b\right)}\left(1-\delta_{n}\right) R_{n},
$$

or, if only terms of the first order in $\Delta$ are retained,

$$
\Lambda_{n} \cong-\Delta\left[1+\frac{n(n+1)}{(\gamma a)^{2}}\right]-Z_{n}
$$

For further analysis, it is also convenient to note that

$$
\hat{I}_{n}^{\prime}(\gamma b) \hat{K}_{n}(\gamma a)-\hat{K}_{n}^{\prime}(\gamma b) \hat{I}_{n}(\gamma a)=N_{n},
$$

and

$$
\hat{I}_{n}(\gamma b) \hat{K}_{n}(\gamma a)-\hat{K}_{n}(\gamma b) \hat{I}_{n}(\gamma a)=Q_{n}
$$




\section{Surface Currents and Input Impedance}

The surface density, $j_{\theta}$, of the currents excited on the surface of the conducting sphere is numerically equal to $H_{\phi}(a, \theta)$, which is the tangential magnetic field. An explicit expression for this quantity is readily found to be

$$
j_{\theta}=H_{\phi}(a, \theta)=\frac{1}{\gamma a} \sum_{n=1}^{\infty} e_{n} \frac{N_{n}+\left(Z_{n} / \eta\right) Q_{n}}{M_{n}+\left(Z_{n} / \eta\right) R_{n}} P_{n}^{\prime}\left(\cos \theta_{0}\right) \mathrm{A} / \mathrm{m},
$$

where $Z_{n}$ and $e_{n}$ are defined by (19) and (29), respectively, while $R_{n}, Q_{n}, N_{n}$, and $M_{n}$ are defined above.

The total input conductance $G$ of the slot may be found from

$$
G=\frac{1}{V_{0}}\left(2 \pi a \sin \theta_{0}\right) \operatorname{Re} H_{\phi}\left(a, \theta_{0}\right),
$$

as indicated in the previous paper [Wait, 1964a] for a similar problem. This quantity is finite since the dielectric sheath is lossless.

Without specifying the conditions of the field within the slot, it is not possible to calculate the total susceptance of the slot. However, a useful quantity, which may be calculated, is the change of the self-impedance $\Delta Z$ which results when the medium external to the surface $r=b$ is changed from free space to plasma. Thus

$$
\Delta Z=\frac{2 \pi a \sin \theta_{0}}{V_{0}}\left[H_{\phi}\left(a, \theta_{0}\right)-\tilde{H}_{\phi}\left(a, \theta_{0}\right)\right]
$$

where $\tilde{H}_{\phi}(a, \theta)$ has the same form as $(54)$ if $Z_{n}$ is replaced by $\tilde{Z}_{n}$, which is defined by

$$
\widetilde{Z}_{n}=-\eta_{0} \frac{\hat{K}_{n}^{\prime}\left(\gamma_{0} b\right)}{\hat{K}_{n}\left(\gamma_{0} b\right)},
$$

where $\eta_{0}=\left(\mu_{0} / \epsilon_{0}\right)^{\frac{1}{2}}$ and $\gamma_{0}=i\left(\epsilon_{0} \mu_{0}\right)^{\frac{1}{2}} \omega$. The susceptance change $\Delta B$ is simply the imaginary part of $\Delta Z$.

\section{Concluding Remarks}

The formulas developed in this paper should be useful for computing impedance characteristics of a slotted sphere-type antenna with a dielectric sheath. The parameters of the problem involve the electrical size of the sphere, thickness and dielectric constant of the coating; electron density and collision frequency of the plasma, and the effective acoustic or thermal velocity of the electron fluid. It appears that the dielectric coating reduces the relative amount of power being transferred into the acoustic wave.

Part III of this series will contain extensive numerical data illustrating the rather complicated relations between the various parameters.

\section{References}

Wait, J. R. (1964a), Theory of slotted-sphere antenna immersed in a compressible plasma. Part I. Radio Sci. J. Res. NBS/USNC-URSI 68D, No. 10, 1127-1136 (Oct. 1964). Contains extensive references to related work.)

Wait, J. R. (1964b), Radiation from sources immersed in compressible plasma media, Can. J. Phys. (in press).

(Paper 68D10-413) 